\title{
El rastreo y tratamiento agresivo del cáncer de próstata pareciera no reducir la mortalidad por esta enfermedad
}

Natural experiment examining impact of aggressive screening and treatment on prostate cancer mortality in two fixed cohorts from Seattle area and Connecticut. Lu-Yao G et al. BMJ 2002; 325:710-6.

\section{Objetivo}

Determinar si la mortalidad por cáncer de próstata (CP) fue menor en una región (Seattle) que implementó una estrategia agresiva de rastreo y tratamiento que en otra (Connecticut), donde la energía con la que se aplicó el rastreo fue menos intensa.

\section{Diseño}

Experimento natural en el que se compararon los datos epidemiológicos a lo largo de diez años (1987-1997) de dos cohortes fijas: una en Seattle-Puget Sound y otra en Connecticut.

\section{Principales variables evaluadas}

Tasa de rastreo de CP, tratamiento mediante prostatectomía radical y radioterapia externa, y mortalidad específica por CP.

\section{Resultados}

En Seattle, la tasa de indicación de antígeno prostático específico (APE) a los pacientes fue 5,39 veces mayor que en Connecticut (IC 95\%: 4,76-6,11) y la tasa de biopsias indicadas fue 2,20 veces mayor $(1,81-2,68)$. En cuanto a la incidencia de prostatectomías radicales y radioterapias externas, en Seattle las tasas fueron del $2,7 \%$ y el $3,9 \%$ respectivamente, mientras que en Connecticut fueron del $0,5 \%$ y el $3,1 \%$.No hubo diferencias significativas entre la mortalidad específica por CP de Seattle y la de Connecticut.RR: 1,03 (IC95\% 0,95 a 1,11).Ver figura 1.
Figura 1: Mortalidad por CP cada 100.000 personas-año

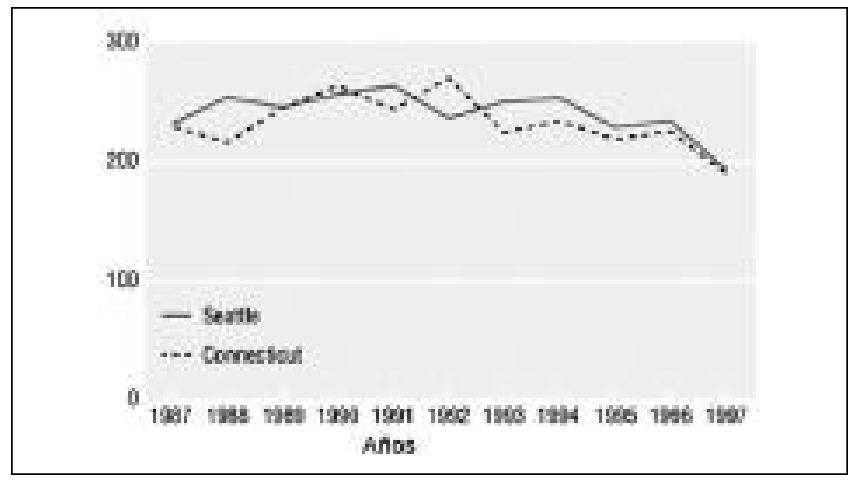

\section{Conclusiones}

Luego de 10 años de seguimiento no se ha podido comprobar que la mortalidad específica por CP sea más baja en una región que utilizó una estrategia más agresiva de rastreo y tratamiento del CP (Seattle) que en otra que utilizó una estrategia menos agresiva (Connecticut).

\section{Comentario}

Este es un estudio interesante para los que todavía consideramos que no tiene mucho sentido hacer rastreo de CP ya que demuestra que lo que nos interesa finalmente: que disminuya la mortalidad por CP no depende de la agresividad del rastreo.Con los resultados de este estudio podríamos dar una charla y decir: "miren, en Seattle rastrearon a un montón de personas, hicieron un montón de prostatectomías radicales y tuvieron, al cabo de diez años, la misma tasa de mortalidad específica por CP que en Connecticut, donde la tasa de rastreo fue cinco veces más baja y hubo menos prostatectomías". Sin embargo, la cosa no es tan sencilla y, en esta misma línea de pensamiento epidemiológico, se publicó un estudio austríaco que demostró lo contrario: la mortalidad por $\mathrm{CP}$ fue menor en una región de Tirol que implementó un programa agresivo de rastreo'. Conclusión. Estamos un poco cansados del eterno dilema acerca de si vale o no la pena hacer rastreo de CP y no vemos la hora de conocer los resultados del ensayos clínicos bien diseñados (aleatorizados, controlados) que se están llevando a cabo para dilucidar este dilema, y cuyas conclusiones conoceremos no antes de $2005^{2}$, ${ }^{3}$.La recomendación con res- pecto a la utilidad de hacer rastreo es, por el momento, de tipo 14 (insuficiente): o sea: incertidumbre, lo que implica, en la práctica en el consultorio, ser honestos con nuestro paciente, explicarle que no sabemos si tiene sentido hacer rastreo y que sea él quien elija qué hacer. Igualmente, esto no es tan fácil de lograr en consultas de veinte minutos o menos, con muchos temas por tratar, y siempre es más sencillo escribir una orden con una rutina que incluya al APE como parte del "control" que explicarle todo esto del dilema del rastreo del CP al paciente.

Conclusiones del comentador: todavía no se ha resuelto el dilema de la utilidad del rastreo de CP y habrá que aguardar los resultados de ensayos en marcha. Hasta entonces, siempre que sea posible, habría que intentar transmitirle esta información al paciente de manera que pueda tomar una decisión acorde a sus propias creencias.

Dr. Esteban Rubinstein [ Unidad de Medicina Familiar.Hospital Italiano de Buenos Aires ]

\section{Referencias:}

1.Bartsch G, Horninger W, Klocker H, Reissigl A, Oberaigner W, Schonitzer D, et al.Prostate cancer mortality after introduction of prostate-specific antigen mass screening in the federal state of Tyrol, Austria. Urology 2001;58:417-424

2.Norlén BJ. Swedish randomized trial of radical prostatectomy versus watchful waiting. Can J Oncol.1994;4(Suppl 1):38-40.

3. Moon TD, Brawer MK, Wilt TJ.Prostate Intervention Versus Observation Trial (PIVOT): a randomized trial comparing radical prostatectomy with palliative expectant management for treatment of clinically localized prostate cancer. PIVOT Planning Committee.J Natl Cancer Inst Monogr. 1995;(19):69-71.

4.R.Harris and K. N.Lohr.Screening for Prostate Cancer:An Update of the Evidence for the U.S. Preventive Services Task Force

Ann Intern Med, December 3, 2002; 137(11): 917 - 929. 